\title{
Pseudotumor cerebri syndrome in childhood: incidence, clinical profile and risk factors in a national prospective population-based cohort study
}

Yim-Yee Matthews ${ }^{1}$, Fiona Dean², Ming J Lim³ ${ }^{3}$ Karen Mclachlan ${ }^{4}$, Alan S Rigby ${ }^{5}$, Guirish A Solanki ${ }^{6}$, Catharine P White ${ }^{7}$, William P Whitehouse ${ }^{8}$, Colin R Kennedy ${ }^{9}$

${ }^{1}$ Paediatric Department, Wrexham Maelor Hospital, Wrexham, UK

${ }^{2}$ Ophthalmology Department, University Hospital Coventry and Warwickshire NHS Trust, Coventry, UK

${ }^{3}$ Children’s Neurosciences, Evelina London Children's Hospital, Guy's and St Thomas' NHS

Foundation Trust, King’s Health Partners Academic Health Science Centre, London, UK

${ }^{4}$ Paediatric Department, University Hospital Coventry and Warwickshire NHS Trust, Coventry, UK

${ }^{5}$ University of Hull, Hull, UK

${ }^{6}$ Department of Paediatric Neurosurgery, Birmingham Children’s Hospital, University of Birmingham, Birmingham, UK.

${ }^{7}$ Department of Child Health, Morriston Hospital, Swansea, UK

${ }^{8}$ School of Medicine, University of Nottingham, Paediatric Neurology, Nottingham University Hospitals NHS Trust, Nottingham, UK

${ }^{9}$ Clinical Neurosciences, Faculty of Medicine, University of Southampton, University Hospital Southampton, Southampton, UK

\section{Correspondence to}

Dr Yim-Yee Matthews, Paediatric Department, Wrexham Maelor Hospital, Croesnewydd Road, Wrexham LL13 7TD, UK; yim-yee.matthews@wales.nhs.uk Telephone: +44 1978291100

Word counts: Abstract (248/250), Manuscript (2875/2500) 


\section{ABSTRACT}

Aim To investigate the epidemiology, clinical profile and risk factors of pseudotumor cerebri syndrome (PTCS) in children aged 1-16 years.

Methods A national prospective population-based cohort study over 25 months. Newly diagnosed PTCS cases notified via British Paediatric Surveillance Unit (BPSU) were ascertained using classical diagnostic criteria and categorised according to 2013 revised diagnostic criteria. We derived national age, sex and weight-specific annual incidence rates and assessed effects of sex and weight category. Results We identified 185 PTCS cases of which 166 also fulfilled revised diagnostic criteria. The national annual incidence (95\% CI) of childhood PTCS aged 1-16 years was 0.71 (0.57- 0.87) per 100,000 population increasing with age and weight to 4.18 and 10.7 per 100,000 in obese $12-15$ year old boys and girls respectively. Incidence rates under 7 years were similar in both sexes. From 7 years onwards, the incidence in girls was double that in boys, but only in overweight (including obese) children. In 12-15 year old children, an estimated 82\% of the incidence of PTCS was attributable to obesity. Two subgroups of PTCS were apparent: 168 (91\%) cases aged from 7 years frequently presented on medication and with headache, and were predominantly female and obese. The remaining 17 (9\%) cases under 7 years often lacked these risk factors and commonly presented with new onset squint.

Conclusions This uniquely largest population-based study of childhood PTCS will inform the design of future intervention studies. It suggests that weight reduction is central to the prevention of PTCS. 


\section{INTRODUCTION}

Pseudotumor cerebri syndrome (PTCS), also known as idiopathic intracranial hypertension, is a rare syndrome of raised intracranial pressure (ICP) first described in $1893 .{ }^{1}$ Its annual incidence in adults and children has been estimated to be $1 \cdot 6-2 \cdot 2^{2-4}$ and $0.5-0.9^{5-7}$ per 100,000 respectively although the latter estimates are based on small or retrospective studies. PTCS has potentially serious morbidities of distressing headache, blindness, ${ }^{89}$ and complications of invasive treatment procedures. Its management remains controversial.

Classical diagnostic criteria for PTCS, in use since $1937,{ }^{10}$ combine the absence of other causes of raised ICP, including mass lesions and cerebral venous sinus thrombosis (CVST), with the presence of three cardinal features: symptoms of raised ICP, papilloedema and elevated opening cerebrospinal fluid (CSF) pressure at lumbar puncture (LP). ${ }^{11}$ These criteria provided the present study case definition (Table 1A). Although CVST excludes the diagnosis of PTCS, the presence of risk factors does not and it is therefore not 'idiopathic'. The fidelity of the clinical diagnosis of PTCS may be reduced first by misdiagnosis of papilloedema when evaluated by clinicians lacking access to specialist ophthalmological assessment ${ }^{12}$ and second by estimation of CSF pressure based on brief recording of lumbar CSF opening pressure. This varies with posture, movement, emotion, anaesthetics and hypercapnia, and may not accurately reflect mean CSF pressure. ${ }^{13}$

An increase in the upper normal threshold of lumbar CSF opening pressure from $20 \mathrm{~cm}$ to $25 \mathrm{~cm}$ CSF in non-obese children and $28 \mathrm{~cm}$ in children who are either obese or sedated for their LP has been proposed. ${ }^{14}$ Furthermore, specific (but insensitive) neuroradiological markers of raised ICP have been established. ${ }^{15-19}$ In 2013, Friedman et al. published revised diagnostic criteria for PTCS that incorporated these changes but did not include symptoms of raised ICP (Table 1B). ${ }^{20}$ The revised criteria categorise PTCS as 'definite’ (elevated CSF pressure and either papilloedema or abducens nerve palsy), 'probable' (papilloedema but normal CSF pressure) or 'suggestive of PTCS' (lacking papilloedema and abducens nerve palsy but manifesting elevated CSF pressure plus at least three valid neuroimaging markers of raised ICP). ${ }^{20}$ 
This study aimed to establish the incidence of childhood PTCS reliably, quantify the impact of known risk factors and identify clinical subgroups.

\section{METHODS}

We ascertained newly diagnosed PTCS cases aged 1-16 years presenting over a 25-month period (August 2007-October 2009) to general or specialised paediatricians in the UK or Ireland using the monthly reporting scheme of the British Paediatric Surveillance Unit (BPSU). ${ }^{21}$ Notifying clinicians completed questionnaires detailing demographics, referral source, presentation, prior medications and medical conditions, and anthropometric, ophthalmological, CSF and neuroimaging data. YYM assessed jointly with at least one co-author whether the submitted data fulfilled the study case definition (Table 1A). The validity of the diagnosis was checked with the notifying clinician one year after diagnosis. Data collection concluded in July 2013. Each valid PTCS case was then categorised according to the subsequently published revised diagnostic criteria (Table 1B).

CSF opening pressure reported above a recorded value was rounded up to the nearest integer (e.g. ‘ $>40 \mathrm{~cm}$ ' became ‘41cm'). The age- and sex-specific annual incidence for eligible PTCS cases was determined from contemporary population estimates 2223 and evaluated in three age categories: 1-6, 7-11 and 12-16 years. The choice of these three age categories was based on previous reports of variation of incidence rates with age and puberty status. Age-specific incidences for each year of age within each age band did not differ significantly from each other (data not shown).

Participants' growth data were expressed as z-scores of body mass index (or weight, when height was unavailable) in three weight categories: non-overweight (z-score $<1.04=<85^{\text {th }}$ percentile), overweight (excluding obese) ( $\mathrm{z}$-score $\geq 1.04$ to $<1.64=\geq 85^{\text {th }}$ to $<95^{\text {th }}$ percentile) and obese ( $\mathrm{z}$-score $\geq 1.64=\geq 95^{\text {th }}$ percentile) relative to the sex and age specific 1996 British Growth reference population. ${ }^{24}$ The weightspecific annual incidence of PTCS for cases aged 4-6, 7-11 and 12-15 years was derived relative to the prevalence of these weight categories in national health surveys that were available for those age ranges. $^{25}$ The UK and Republic of Ireland population age 1-16 years during the survey was 12,451,742 of which 6,375,886 were male. The population aged 4-15 years included 1,604,499 males among 
3,003,247 overweight children. Age-, sex- and weight-specific annual incidence rates were calculated with 95\% Confidence Intervals (CI) based on the Poisson distribution.

Continuous data were summarised as median values with interquartile ranges (IQRs) and categorical data as $n(\%)$. Homogeneity of variance was assessed with the Levene F test. Case-wise deletion was performed for missing data. Groups were compared by Mann-Whitney U or Kruskal-Wallis tests and Fisher's Exact test for continuous and categorical data respectively. The impact of the known risk factors for PTCS was expressed as Relative Risk (RR), Population Attributable Risk (PAR) and Percentage PAR (\%PAR). IBM SPSS Statistics 21 was used with two-tailed statistical testing at $\mathrm{p}<0 \cdot 05$.

This study was approved by the East London \& The City Research Ethics Committee, the Patient Information Advisory Group and the North Wales NHS Research \& Development.

\section{RESULTS}

Of 379 notifications, 185 (49\%) fulfilled the study case definition for newly diagnosed PTCS (Figure 1). Of these, $124 / 185$ (67\%) were girls, $167 / 185$ (90\%) were of white ethnicity and 157/185 (85\%) were directly referred to general paediatricians. The mean case accrual rate and percentage of cases relative to total notifications for each 6-month period remained steady throughout the 25-month survey at 46 (range 44-48) cases and 50\% (range 42-57) respectively. The diagnosis remained valid in all 163/185 (88\%) eligible cases for whom one year follow-up data were available (Figure 1).

\section{Incidence by age, sex and weight category}

The annual incidence of PTCS (95\% CI) aged 1-16 years was $0.71(0.57-0.87)$ per 100,000 population. The age-specific incidence increased from 0.17 , to 0.75 and then to 1.32 per 100,000 in 1-6, 7-11 and 12-16 year olds respectively (Appendix Figure e1). Incidence rates in girls vs boys were 0.19 vs 0.16 , 1.06 vs 0.46 and 1.85 vs 0.82 per 100,000 in these respective age categories (Appendix Figure e1). Annual incidence per 100,000 obese population increased with age in 4-6, 7-11 and 12-15 year olds from 0.45 to 1.24 and then to 4.18 in boys and from 0.56 to 3.44 and then to 10.7 in girls (Appendix 
Figure e1). The incidence remained low at 0.18 to 0.42 per 100,000 among non-overweight boys and girls throughout the 4-15 year age range (Appendix Figure e1).

Weight and height measurements were available in $100 \%$ and $94 \%$ of cases respectively. Twenty-one (11\%) cases were overweight and 120/185 (65\%) were obese (Figure 2). In both sexes, the percentage of obese cases increased significantly with age $(\mathrm{p}<0.01)$ across the age categories from $41 \%(7 / 17)$, to $50 \%(29 / 58)$ and then to $76 \%(84 / 110)(\mathrm{p}<0.001)$. The relative risks $(\mathrm{RR})$ of PTCS associated with obesity relative to non-overweight children of the same age and sex were 3.6 and 8.1 in 7-11 year old boys and girls respectively and 23.3 and 26.2 in 12-15 year old boys and girls respectively (Figure 3). Among overweight (including obese) children, the RR for PTCS in girls relative to boys was 3.7 in 711 year olds and 2.4 in 12-15 year olds (Figure 3 ).

\section{Key diagnostic and neurological features}

The triad of raised ICP symptoms, papilloedema and elevated CSF pressure, was seen in 83\% (153/185) and 73\% (136/185) of those using the study and higher revised ${ }^{14}$ CSF pressure threshold criteria respectively (Figure 1). Papilloedema, symptoms of raised ICP and elevated CSF pressure were absent in 11\% (20/185), 4\% (8/185) and 2\% (4/185) respectively or, using the revised CSF pressure threshold ${ }^{14} 9 \%$ (16/185), 4\% (7/185) and 11\% (21/185) of cases (Figure 1). There were no differences between cases with and without any one of the three diagnostic cardinal features with respect to other presenting features, sex, age and weight categories, or other risk factors.

Headache was present in 161/185 (87\%) cases. Diplopia, blurring of vision or transient visual loss were reported in 87/161 (54\%) with and 15/24 (62\%) cases without headache. One or more other neurological features were reported in 70/185 (38\%) cases (32 abducens nerve palsy, 19 new presentation of squint, 17 dizziness, 12 photophobia, 8 neck stiffness/pain, 5 ataxia/falling, 4 tinnitus, 3 vertigo, 2 each of facial weakness, torticollis, nystagmus, vertigo, paraesthesia and pain behind eyes).

Papilloedema was reported in 165/185 (89\%), was unilateral in 13/185 (7\%) and was detected incidentally in 5/185 (3\%) asymptomatic cases. Ophthalmologists confirmed its presence in all but five cases and also affirmed its absence in the 20/185 (11\%) cases without papilloedema. Optic nerve head 
drusen (ONHD) was co-existent with papilloedema in 8/185 (4\%), absent in 134/185 (72\%), and uncertain in 27/185 (15\%) cases. Of 32/185 (17\%) cases with abducens nerve palsy, 30/32 (94\%) were accompanied by papilloedema.

New presentation of decreased visual acuity (DVA) and/or restricted visual fields (RVF) at diagnosis were reported in 61/185 (33\%) of the 182/185 (98\%) cases whose vision was assessed. Seventeen had DVA, 32 RVF, 11 both DVA and RVF and 1 unspecified visual defects. Of those with a visual deficit, 54/61 (89\%) had papilloedema. No differences were detected in the proportion of visual deficit between sexes, age or weight categories.

\section{Comparison of cases fulfilling study and revised diagnostic criteria}

Of the 185 valid study cases, PTCS diagnosis was classified on revised criteria as ‘definite’ in 144 (78\%), 'probable' in 22 (12\%), not present in 4 (2\%) cases and 'undetermined' in 15 (8\%) in whom the presence (or not) of neuroimaging criteria required for the 'suggestive of PTCS' category had not been assessed (Figure 1). The 166 ‘definite’ or 'probable’ PTCS cases did not differ from the other 19 cases with respect to age, sex, weight category, other associations or clinical features other than papilloedema.

\section{Relation of neurological features to age and weight}

The report of headache increased from 41\% (7/17) of 1-6 year olds to 91\% (53/58) of 7-11 year olds and stabilised at $90 \%(99 / 110)$ of $12-15$ year olds $(\mathrm{p}<0.001$, Figure 4$)$. The incidence of blurring of vision also increased across these age categories from $24 \%(4 / 17)$ to $28 \%(16 / 58)$ then to 53\% (58/110) $(p=0.002$, Figure 4). Conversely, there was a reduction in new onset squint with successive age categories from $29 \%(5 / 17)$ to $9 \%(5 / 58)$ then to $8 \%(9 / 110)$ respectively $(\mathrm{p}=0.024)$ and of transient visual loss from $35 \%(6 / 17)$ to $10 \%(6 / 58)$ then to $9 \%(10 / 110)$ respectively $(\mathrm{p}=0.007)$ (Figure 4$)$.

\section{Risk factors for PTCS and their relation to age and sex}

Ninety-three (50\%) cases had one and 61 (33\%) had more than one recognised risk factor at diagnosis (Appendix Table e1). Overweight or obesity was the only risk factor in 87/185 (47\%) and was accompanied by other risk factors in 54/185 (29\%) cases (Appendix Table e1). Among 46/185 (25\%) 
cases on medication, 16 (9\%) had a medical condition and girls were overrepresented (40/124 or 32\% vs $6 / 61$ or $10 \%, \mathrm{p}=0.001)$. A further $21(11 \%)$ cases had a medical condition without associated medication. The commonest reported medical conditions were migraine, in 18/185 (10\%), and antibiotic use for acne, in 10/185 (5\%). The percentage of cases with risk factors increased with successive age categories from $59 \%(10 / 17)$ to $76 \%(44 / 58)$ then to $91 \%(100 / 110)(p=0.001)$.

\section{Neuroimaging and CSF findings}

All cases had cranial neuroimaging and this included venography in 111/185 (60\%). Those with and without venography did not differ with respect to age, sex, weight, clinical features or other risk factors. At least one neuroimaging criteria of raised ICP ${ }^{20}$ (Table 1B) was reported in 10/185 (5\%) cases, all of whom had papilloedema but four lacked elevated CSF pressure on revised criteria.

The median CSF opening pressure was 35.5cm CSF (range 12.0-74.0). It did not differ significantly between sexes or age categories, cases with and without headache or with and without papilloedema but was significantly higher in obese than other weight categories cases $(p=0.004)$ and in the 82 cases whose LPs were undertaken with general anaesthesia (GA) compared to the 100 cases undertaken without GA ( $p=0.001$ ) (Appendix Figure e2). However, the percentage of cases whose LP required GA did not differ between weight categories.

\section{DISCUSSION}

This is the largest study of childhood PTCS to date and shows that the incidence increases eightfold from that in 1-6 years to that in 12-16 years and the numbers of girls and boys changes from being equal under the age of seven to two girls for each boy thereafter. These shifts were linked to obesity accounted for over four-fifths of the increased incidence among those aged 12-15 years. Even with the upwardly revised CSF pressure threshold, ${ }^{14}$ nearly three-quarter of cases had a classic triad of raised ICP symptoms, papilloedema and elevated CSF pressure and the clinical profile did not differ in other ways between cases with and without any one of the three cardinal features.

On revised diagnostic criteria ${ }^{20} 90 \%$ of cases captured by the study definition were classified as ‘definite’ or ‘probable’ PTCS and did not differ clinically from the remaining $10 \%$ except in 
manifesting papilloedema. This is consistent with PTCS being a single entity that includes either papilloedema or elevated CSF pressure or both. While PTCS is thus distinct, the findings on magnetic resonance venography and CSF haemodynamics are similar to those in the 'slit ventricle syndrome' of elevated CSF pressure. ${ }^{26}$ Slit ventricle syndrome is defined in patients with symptoms of raised ICP due to intraventricular shunt malfunction with slit-like radiological appearance of small ventricles. This usually arises when intermittent and functional shunt obstruction is associated with decreased compliance of the tissues surrounding the cerebral ventricles.

Although 90\% were of white ethnicity, PTCS were reported in all ethnic groups. We believe this truly represents our nationwide paediatric practice. A smaller series of cases in California found similar relationships to those seen in the present study between age, sex and overweight, but also observed a significant increase in the risk of PTCS in non-Hispanic white children relative to that in Hispanic white or Asian/Pacific Island chldren. ${ }^{27}$

Cases under 7 years were less likely to have identifiable risk factors or to complain of headache or blurred vision but more likely to report transient visual loss and manifest squint. Their immature language skills may account for the differences in expressing symptomatology subjectively. However, their commoner squint presentation and the lack of association with sex, overweight or medication use may reflect late presentation and/or a different pathophysiology. Dysregulation of glucocorticoid signalling can alter ICP dynamics and cause elevation of ICP in obese individuals ${ }^{28}$ whereas the mechanisms of PTCS in non-overweight younger children remain unclear.

A strength of this study is that it is potentially free from intentional or unintentional bias: the robust BPSU scheme for reporting cases prospectively has $94 \%$ reporting rate ${ }^{29}$ by unselected general and specialised paediatricians across the UK and Ireland whose rate of reporting and case detection was consistent throughout the survey period. It is very improbable that any confirmed childhood PTCS cases were managed without the involvement of a paediatrician and the study case definition was inclusive so this study is likely to have captured the full spectrum of newly diagnosed PTCS. Nevertheless, missed or unreported cases are inevitable with the voluntary BPSU reporting scheme and our estimates should be regarded as an estimate of the minimum incidence rate. We cannot exclude the 
possibility that a higher proportion of all overweight cases were ascertained due to paediatricians having a higher index of suspicion of the disorder in those children. We did not have an alternative source of information from which to estimate incidence or confirm completeness of ascertainment using capture/recapture analysis.

Other strengths of the study were the avoidance of diagnostic error by evaluation of suspected papilloedema and drusen (ONHD) by ophthalmologists, submission of weight and height data, documentation of GA use at LP, confirmation of the diagnosis one year later, neuroimaging including exclusion of CVST which may be clinically indistinguishable from PTCS. ${ }^{30}{ }^{31}$ Limitations of the study include the lack of standardisation in the method of assessment of opening CSF pressure and the failure of neuroradiological reports, in most cases, to give specific consideration to potential specific neuroradiological markers of raised ICP thus reducing the number of cases that could be categorised as 'suggestive of PTCS'.

Over-diagnosis of PTCS relative to the study case definition was present in $13 \%$ of notifications. This carries a potential risk of harm suggesting the need for greater awareness of the diagnostic criteria among paediatricians. The higher CSF opening pressures seen in children that were obese or had GA for LP support the upwards revision of the diagnostic CSF pressure threshold in these children.

In summary, this study provides three important findings relating to childhood PTCS. First, it suggests that it is a unitary syndrome and describes its epidemiology reliably. These observations should be generalisable to populations of similar ethnicity and weight profile. Second, it established female sex and obesity as the dominant risk factors from age 7 years. Third, it shows that children aged under 7 years do not show these risk factors and may present somewhat differently. The age, sex and weightspecific PTCS incidence data will help investigators to estimate the size of the population required to achieve an adequately powered interventional trial. These data also suggest that the avoidance of overweight and weight reduction may have considerable potential impact as primary and secondary preventive measure for PTCS in children over the age of 7 years. . 
Acknowledgements The authors are very grateful to all the general and specialised paediatricians in the UK and Ireland who contributed to the notification and submission of survey questionnaires data, BPSU assisted in the development of the study protocol and survey questionnaires, to SHINE (Spina Bifida. Hydrocephalus. Information. Networking. Equality, formerly the Association for Spina Bifida and Hydrocephalus) and IIH UK for their support, to Dr Krystyna Matyka for contribution in the study design and case ascertainment, to Dr Jim Turner, Senior Research Fellow, for statistical support and to Mrs Jayne Cooke for administrative support.

Contributors The surveillance study was designed by YYM with key input from KMc and FD in development of study protocol and survey questionnaires. WPW, CRK and CPW provided oversight of the surveillance study conducted by YYM. YYM, FD, KMc, CPW, WPW and CRK evaluated the eligibility of notifications. YYM analysed and interpreted the collected data. ASR advised and provided input in the statistical analysis. YYM did the literature reviews, drafted and revised all versions of the manuscript. CRK together with contributions from all others (WPW, MJL, CPW, FD, KMc, GAS and ASR) critically involved in the data interpretation, revised all versions and approved the final version of the manuscript.

Completing interests MJL receives research grants from Action Medical Research, DES society, GOSH charity, NIHR, MS Society, SPARKS charity and; receives research support grants from the London Clinical Research Network and Evelina Appeal; has received consultation fees from CSL Behring; received travel grants from Merck Serono; and awarded educational grants to organize meetings by Novartis, Biogen Idec, Merck Serono and Bayer. GAS has been an investigator in MPSII clinical trials with Shire, has received speaker’s honorarium and travel support from BioMarin. None declared by all other authors (YYM, FD, KMc, ASR, CPW, WPW \& CRK).

Funding This national survey was supported by the BPSU/RCPCH Sir Peter Tizard Research Bursary. 


\section{What is already known on this topic?}

$>$ Pseudotumor cerebri syndrome (PTCS) is a rare syndrome of intracranial hypertension defined by an evolving diagnostic algorithm, most recently revised in 2013.

> Risk factors for PTCS including female sex and obesity.

> The epidemiology of PTCS in childhood has not been reliably established in a prospective study but is thought to be lower than that in adults.

\section{What this study adds?}

$>$ This prospective survey of all cases in Great Britain and Ireland is the largest study of PTCS in childhood and establishes, for the first time, reliable estimates of age, sex and weight specific annual incidence rates.

$>$ In a few, childhood PTCS occurs prior age of 7 years, often without identifiable risk factors and with some distinctive features in its clinical presentation.

Most childhood PTCS occurs from age 7 years when it is twice as common in girls and progressively commoner with increasing age and degrees of overweight. More than $80 \%$ of PTCS in 12-15 year olds is attributable to obesity. 


\section{REFERENCES}

1 Quincke H. Über meningitis serosa. Sammlung Klinische Vortrage (Innere Medezin 23) 1893;67:655-94.

2 Kesler A, Stolovic N, Bluednikov Y, et al. The incidence of idiopathic intracranial hypertension in Israel from 2005 to 2007: results of a nationwide survey. Eur J Neurol 2014;21:1055-9.

3 Raoof N, Sharrack B, Pepper IM, et al. The incidence and prevalence of idiopathic intracranial hypertension in Sheffield, UK. Eur J Neurol 2011;18:1266-8.

4 Idiculla $\mathrm{T}$, Zachariah G, Br K, et al. The incidence and prevalence of idiopathic intracranial hypertension in south Sharaqiah region, Oman. Oman J Ophthalmol 2013;6:189-92.

5 Gordon K. Pediatric pseudotumor cerebri: descriptive epidemiology. Can J Neurol Sci $1997 ; 24: 219-21$.

6 Tibussek D, Distelmaier F, von Kries R, et al. Pseudotumor cerebri in childhood and adolescence results of a Germany-wide ESPED-survey. Klin Padiatr 2013;225:81-5.

7 Bursztyn LL, Sharan S, Walsh L, et al. Has rising pediatric obesity increased the incidence of idiopathic intracranial hypertension in children? Can J Ophthalmol 2014;49:87-91.

8 Best J, Silvestri G, Burton B, et al. The incidence of blindness due to idiopathic intracranial hypertension in the UK. Open Ophthalmol J 2013;7:26-9.

9 Wall M, George D. Idiopathic intracranial hypertension. A prospective study of 50 patients. Brain $1991 ; 114: 155-80$.

10 Dandy WE. Intracranial pressure without brain tumor: diagnosis and treatment. Ann Surg 1937;106:492-513.

11 Corbett JJ, Mehta MP. Cerebrospinal fluid pressure in normal obese subjects and patients with pseudotumor cerebri. Neurology 1983;33:1386-8.

12 Sinclair AJ, Burdon MA, Nightingale PG, et al. Rating papilloedema: an evaluation of the Frisen classification in idiopathic intracranial hypertension. J Neurol 2012;259:1406-12.

13 Matthews YY. Drugs used in childhood idiopathic or benign intracranial hypertension. Arch Dis Child Educ Pract Ed 2008;93:19-25.

14 Avery RA, Shah SS, Licht DJ, et al. Reference range for cerebrospinal fluid opening pressure in children. N Engl J Med 2010;363:891-3. 
15 Brodsky MC, Vaphiades M. Magnetic resonance imaging in pseudotumor cerebri. Ophthalmology 1998;105:1686-93.

16 Agid R, Farb RI, Willinsky RA, et al. Idiopathic intracranial hypertension: the validity of crosssectional neuroimaging signs. Neuroradiology 2006;48:521-7.

17 Lim MJ, Pushparajah K, Jan W, et al. Magnetic resonance imaging changes in idiopathic intracranial hypertension in children. J Child Neurol 2010;25:294-9.

18 Maralani PJ, Hassanlou M, Torres C, et al. Accuracy of brain imaging in the diagnosis of idiopathic intracranial hypertension. Clin Radiol 2012;67:656-63.

19 Hoffmann J, Huppertz HJ, Schmidt C, et al. Morphometric and volumetric MRI changes in idiopathic intracranial hypertension. Cephalalgia 2013;33:1075-84.

20 Friedman DI, Liu GT, Digre KB. Revised diagnostic criteria for the pseudotumor cerebri syndrome in adults and children. Neurology 2013;81:1159-65.

21 Knowles RL, Friend H, Lynn R, et al. Surveillance of rare diseases: a public health evaluation of the British Paediatric Surveillance Unit. J Public Health (Oxf) 2012;34:279-86.

22 Office for National Statistics. Population Estimates Unit. Mid-2009 Population estimates for UK, England and Wales, Scotland and Northern Ireland: Table 1 UK; estimated resident population by single year of age and sex.

https://www.ons.gov.uk/peoplepopulationandcommunity/populationandmigration/populationestim ates/datasets/populationestimatesforukenglandandwalesscotlandandnorthernireland (accessed 21 Sept 2013).

23 Central Statistics Office. Census 2006 principal demographic results: Table 9 Persons, males and females classified by single year of age. Ireland: Stationery Office, 2007. http://www.cso.ie/en/media/csoie/census/documents/Amended_Final_Principal_Demographic_Res ults_2006.pdf (accessed 21 Sept 2013).

24 Cole TJ, Freeman JV, Preece MA. British 1990 growth reference centiles for weight, height, body mass index and head circumference fitted by maximum penalized likelihood. Stat Med $1998 ; 17: 407-29$.

25 National Obesity Observatory. International comparisons of obesity prevalence. 2009. http://www.noo.org.uk/uploads/doc799_2_International_Comparisons_Obesity_Prevalen.ce2.pdf (accessed 26 Apr 2015). 
26 Bateman GA. Hypertensive slit ventricle syndrome: pseudotumor cerebri with a malfunctioning shunt? J Neurosurg 2013;119:1503-10.

27 Brara SM, Koebnick C, Porter AH, et al. Pediatric idiopathic intracranial hypertension and extreme childhood obesity. J Pediatr 2012 Oct;161(4):602-7.

28 Sinclair AJ, Walker EA, Burdon MA, et al. Cerebrospinal fluid corticosteroid levels and cortisol metabolism in patients with idiopathic intracranial hypertension: a link between 11beta-HSD1 and intracranial pressure regulation? J Clin Endocrinol Metab 2010;95:5348-56.

29 British Paediatric Surveillance Unit, Royal College of Paediatrics and Child Health. BPSU 23rd Annual Report 2008-2009. Scientific co-ordinator’s yearly review of activities. p.46. http://www.rcpch.ac.uk/system/files/protected/page/BPSU\%20complete\%20for\%20web.pdf

30 Higgins JN, Gillard JH, Owler BK, et al. MR venography in idiopathic intracranial hypertension: unappreciated and misunderstood. J Neurol Neurosurg Psychiatry 2004;75:621-5.

31 Lin A, Foroozan R, Danesh-Meyer HV, et al. Occurrence of cerebral venous sinus thrombosis in patients with presumed idiopathic intracranial hypertension. Ophthalmology 2006;113:2281-4. 


\section{Table 1 Diagnostic criteria for pseudotumor cerebri syndrome in children}

\section{A: Classical criteria (study case definition) \\ B: Proposed revised diagnostic criteria (adapted from Friedman et al 201320)}

\section{All 3 essential criteria}

1. Normal conscious level

2. No structural cause for clinical features of raised ICP such as ventricular dilatation, cerebral or vascular lesion or CVST on cranial neuroimaging and/or MR/CT venography

3. Normal CSF microscopy and biochemistry

\section{AND}

\section{at least 2 of the following 3 key features:}

1. Symptoms of raised ICP: headache, nausea, vomiting or irritability and/or specific visual symptoms of diplopia, blurring of vision or transient visual loss

2. Papilloedema (bilateral or unilateral)

3. Elevated LP opening CSF pressure*

\section{All 3 essential criteria}

1. Normal neurological examination except for specified cranial nerve palsies

MR or contrast-enhanced CT neuroimaging:

a. For typical obese female patients: normal brain parenchyma and no evidence of hydrocephalus, mass, structural lesion or abnormal meningeal enhancement

b. For atypical non-obese, prepubertal or patients at high risk for CVST: neuroimaging as for typical obese female patients but also exclusion of CVST by venography

3. Normal CSF microscopy and biochemistry

\section{AND}

\section{for 'Definite' diagnosis}

Either presence of both papilloedema and elevated CSF opening pressure**

Or if no papilloedema, presence of abducens nerve palsy and elevated CSF opening pressure**

\section{for 'Probable' diagnosis}

Presence of papilloedema but no elevated CSF opening pressure**

\section{for 'Suggestive' of diagnosis}

If absence of both papilloedema and abducens nerve palsy but presence of elevated CSF opening pressure** and at least 3 out of 4 neuroimaging criteria i.e. empty sella,

flattening of the posterior aspect of the globe, transverse venous sinus stenosis and

distension of the peri-optic subarachnoid space with or without a tortuous optic nerve

ICP, intracranial pressure; CVST, cerebral venous sinus thrombosis; MR, Magnetic Resonance; CT, Computed Tomography; LP, lumbar puncture; CSF, cerebrospinal fluid.

$*>20 \mathrm{~cm}$ CSF based on published consensus for children i.e. the lower value of the upper normal limits in adults ${ }^{11}$

$* * \geq 25 \mathrm{~cm}$ CSF in non-obese and unsedated child or $\geq 28 \mathrm{~cm}$ CSF if obese and/or sedated for $\mathrm{LP}^{14}$ 
Figure 1

\begin{tabular}{|c|c|c|c|c|c|}
\hline \multicolumn{3}{|c|}{ Total notifications of newly diagnosed PTCS cases 379} & \multicolumn{3}{|c|}{ Details of 102 excluded notifications: } \\
\hline Excluded notifications (full details on the right) & \multicolumn{2}{|l|}{$102(27 \%)$} & 36 Not meeting study case definition & \multicolumn{2}{|l|}{4 CVST } \\
\hline No questionnaires returned (included 3 duplicates) & \multicolumn{2}{|l|}{$46(12 \%)$} & 29 Historic cases & \multicolumn{2}{|c|}{4 Optic nerve head drusen } \\
\hline Duplicates of eligible PTCS cases & \multicolumn{2}{|l|}{$46(12 \%)$} & 15 Reporting error & \multicolumn{2}{|c|}{1 Optic neuritis } \\
\hline $\begin{array}{r}\text { Eligible PTCS cases that fulfilled study case } \\
\text { definition (Table 1A) }\end{array}$ & \multicolumn{2}{|l|}{185 (49\%) } & \multicolumn{3}{|c|}{$\begin{array}{c}13 \text { Duplicates: } 7 \text { not meeting study case definition, } 3 \text { historic cases } \\
\text { and } 3 \text { reporting error }\end{array}$} \\
\hline \multicolumn{3}{|c|}{185 cases fulfilling study case definition of PTCS (Table 1A) } & \multirow{2}{*}{\multicolumn{3}{|c|}{185 cases fulfilling study case definition of PTCS (Table 1A) }} \\
\hline \multirow[b]{2}{*}{ Categorisation by cardinal features of PTCS } & \multicolumn{2}{|c|}{$\begin{array}{l}\begin{array}{l}\text { CSF pressure relative to } \\
\text { threshold level }\end{array} \\
\end{array}$} & & & \\
\hline & $\begin{array}{l}\text { study } \\
\text { threshold a } \\
\text { N (\%) }\end{array}$ & $\begin{array}{l}\text { revised } \\
\text { threshold } \\
\text { N }(\%)\end{array}$ & \multicolumn{2}{|c|}{$\begin{array}{l}\text { Categorisation by revised diagnostic criteria } \\
\text { (Table 1B) }\end{array}$} & $\mathbf{N}(\%)$ \\
\hline $\begin{array}{l}\text { Papilloedema, symptoms of raised ICP \& elevation } \\
\text { of CSF pressure }\end{array}$ & $153(83 \%)$ & $136(73 \%)$ & \multicolumn{2}{|c|}{$\begin{array}{l}\text { Definite PTCS: papilloedema or abducens nerve } \\
\text { palsy* \& elevation of CSF pressure }\end{array}$} & $144(78 \%)$ \\
\hline $\begin{array}{l}\text { Symptoms of raised ICP \& elevation of CSF } \\
\text { pressure, no papilloedema }\end{array}$ & $20(11 \%)$ & $16(9 \%)$ & \multicolumn{2}{|c|}{$\begin{array}{l}\text { Probable PTCS: papilloedema, no elevation of CSF } \\
\text { pressure }\end{array}$} & $22(12 \%)$ \\
\hline $\begin{array}{l}\text { Papilloedema \& elevation of CSF pressure, no } \\
\text { symptoms of raised ICP }\end{array}$ & $8(4 \%)$ & $7(4 \%)$ & \multirow{2}{*}{\multicolumn{2}{|c|}{$\begin{array}{l}\text { Suggestive of PTCS: elevation of CSF pressure, no } \\
\text { papilloedema nor abducens nerve palsy. This } \\
\text { category was undetermined as neuroimaging data } \\
\text { incomplete }\end{array}$}} & \multirow{2}{*}{$15(8 \%)$} \\
\hline $\begin{array}{l}\text { Papilloedema \& symptoms of raised ICP, no } \\
\text { elevation of CSF pressure }\end{array}$ & $4(2 \%)$ & $21(11 \%)$ & & & \\
\hline $\begin{array}{l}\text { Papilloedema, no symptoms of raised ICP nor } \\
\text { elevation of CSF pressure }\end{array}$ & 0 & 5 (3\%) & \multicolumn{2}{|l|}{ Not PTCS on revised criteria } & $4(2 \%)$ \\
\hline
\end{tabular}

Follow-up data at 1 year post-diagnosis of PTCS

Confirmed PTCS diagnosis remained valid 163 (88\%)

Reported lost to follow-up 8 (4\%)

No information available $14(8 \%)$ 
Figure 2

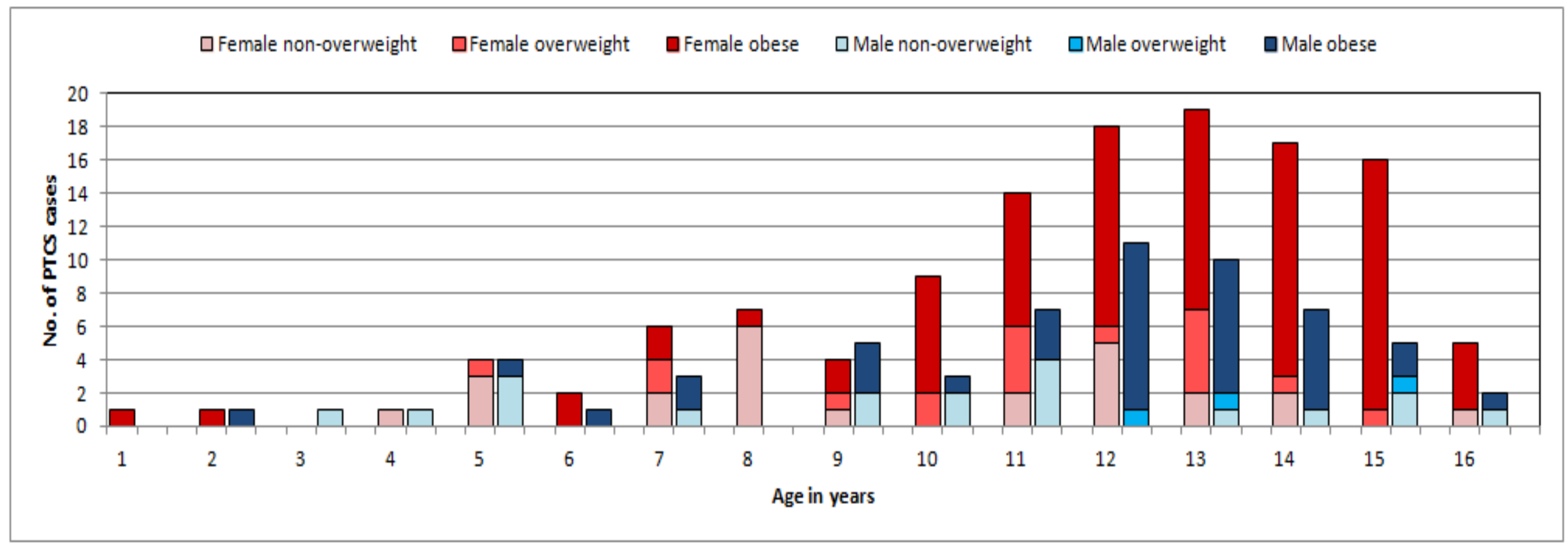


Figure 3
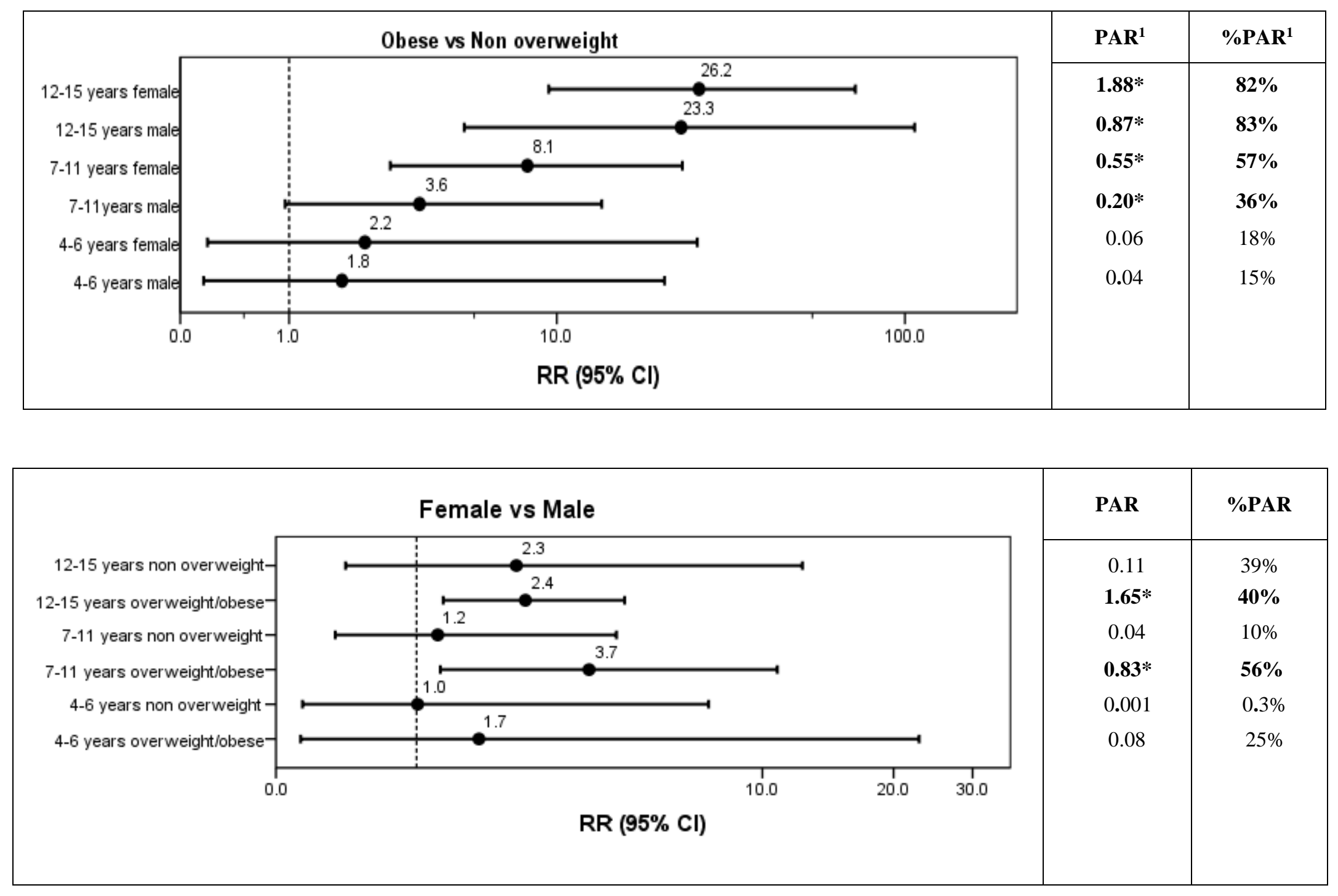


\section{Figure 4}

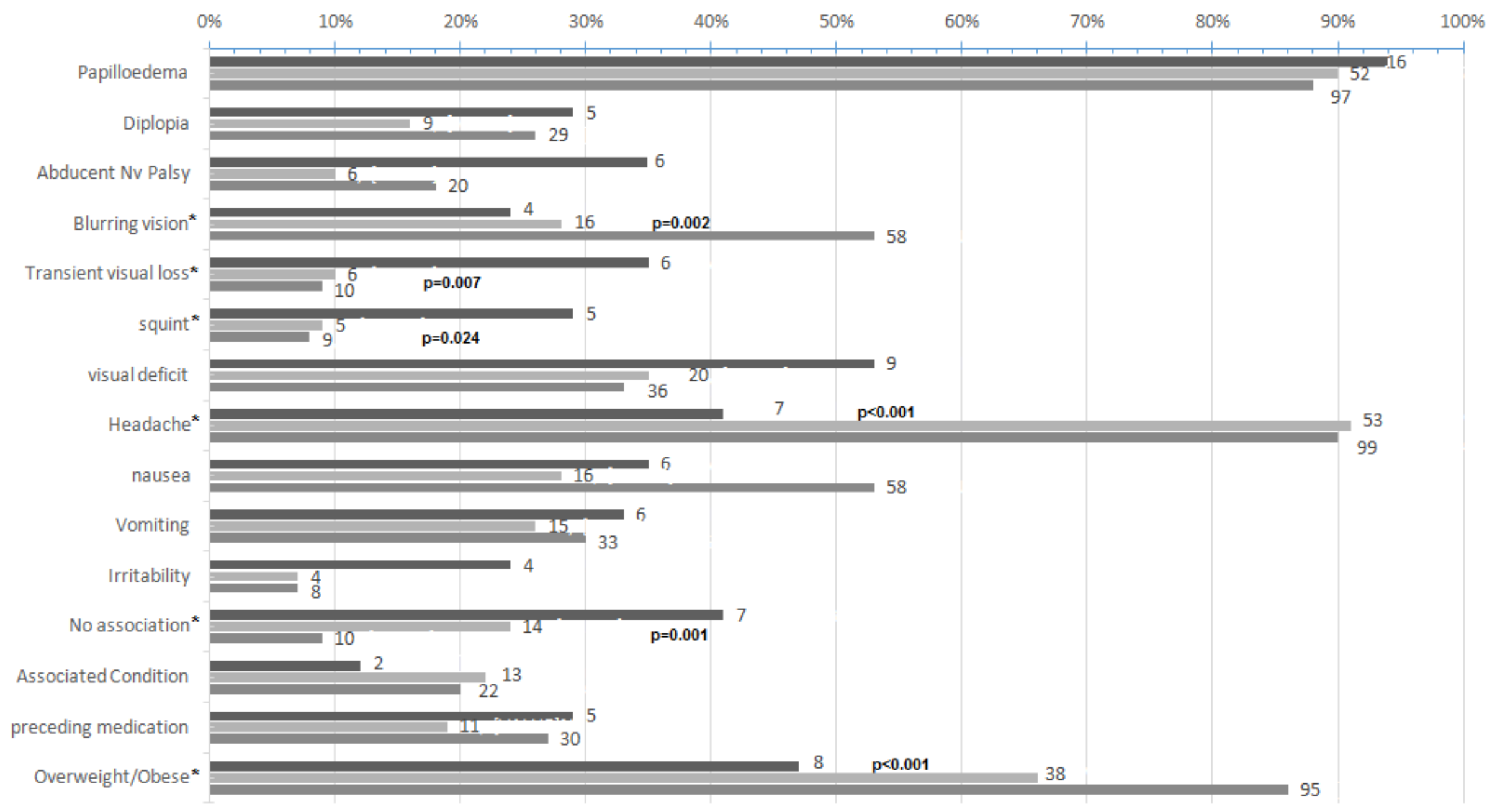

\% with feature aged 1-6 yrs $(n=17)$

\% with feature aged 7-11 yrs ( $n=58)$

— with feature aged $12-16$ yrs $(n=110)$ 


\section{FIGURE LEGENDS}

\section{Figure 1}

\section{Notifications and cases fulfilling diagnostic criteria for pseudotumor cerebri syndrome}

CVST Cerebral Venous Sinus Thrombosis

a: $>20 \mathrm{~cm}$ of CSF. ${ }^{11}$

b: $\geq 25 \mathrm{~cm} \mathrm{CSF}$ in non-obese and unsedated child; $\geq 28 \mathrm{~cm} \mathrm{CSF} \mathrm{if} \mathrm{obese} \mathrm{and/or} \mathrm{sedated} \mathrm{for} \mathrm{LP.}{ }^{14}$

* One definite PTCS case without papilloedema had abducens nerve palsy \& elevated CSF pressure

\section{Figure 2}

\section{Number of cases of pseudotumor cerebri syndrome by age, sex and weight category}

Non overweight: BMI or weight Z-score $<1.04=<85^{\text {th }}$ percentile

Overweight: $\quad$ BMI or weight Z-score $\geq 1.04$ to $<1.64=\geq 85^{\text {th }}$ to $<95^{\text {th }}$ percentile

Obese: $\quad$ BMI or weight Z-score $\geq 1.64=\geq 95^{\text {th }}$ percentile

\section{Figure 3}

\section{Risk of pseudotumor cerebri syndrome associated with obesity and female sex by age}

RR, Relative Risk; error bar, 95\% confidence interval (CI); PAR, Population Attributable Risk.

* the observed RR is statistically significant i.e. 95\% CI does not include unity.

Upper panel: Association between obesity and incidence of pseudotumor cerebri syndrome by sex and age category.

RR of PTCS in obese versus non-overweight children is the ratio of the annual PTCS incidences in obese and non-overweight children, adjusted for age category and sex.

PAR of obesity = annual PTCS incidences in obese and non-overweight children - annual PTCS incidence in non-overweight children

\%PAR of obesity $=($ PAR of obesity x 100)/annual PTCS incidence in obese and non-overweight children.

Lower panel: Association between female sex and incidence of pseudotumor cerebri syndrome by age and weight categories RR in girls versus boys is the ratio of annual PTCS incidence rates in girls and boys, adjusted for age and weight categories. PAR of female sex = annual PTCS incidence in all child population - annual PTCS incidence in boys

\%PAR of female sex $=($ PAR of female sex $x$ 100)/annual PTCS incidence of the total child population

\section{Figure 4}

Number and percentage of neurological features and risk factors of childhood pseudotumor cerebri by age category

$* \mathrm{p}<0.05$ 